\title{
Expression of SLC17A9 in hepatocellular carcinoma and its clinical significance
}

\author{
JINGDONG WU*, YONGFEI YANG ${ }^{*}$ and JIANSHENG SONG \\ Department of Emergency Surgery, The Central Hospital of Wuhan, Tongji Medical College, \\ Huazhong University of Science and Technology, Wuhan, Hebei 430010, P.R. China
}

Received January 16, 2020; Accepted June 24, 2020

DOI: $10.3892 / \mathrm{ol} .2020 .12043$

\begin{abstract}
The present study aimed to investigate the expression and clinical significance of solute carrier family 17 member 9 (SLC17A9) in cancer tissues of patients with hepatocellular carcinoma (HCC). Ninety-eight patients with HCC were admitted to our hospital from January 2010 to December 2014. Their clinical data was retrospectively analyzed. The expression of SLC17A9 in HCC cancer tissues was detected by immunohistochemistry. Kaplan-Meier curve, log-rank test and Cox proportional hazard model analysis were used to analyze the tumor-free survival rate and overall survival rate. SLC17A9 expression was associated with Edmondson grade $(\mathrm{P}=0.04)$ and distant metastasis $(\mathrm{P}=0.03)$. The tumor-free survival $(\mathrm{P}=0.03)$ and overall survival $(\mathrm{P}=0.01)$ of SLC17A9-high expression patients were significantly lower than those in SLC17A9-low expression patients. Multivariate analysis revealed that SLC17A9 expression (HR, 0.77; 95\% CI, 0.27-2.47, $\mathrm{P}=0.02$ ) was an independent risk factor for tumor-free survival in patients with HCC, and the expression of SLC17A9 (HR, 1.81; 95\% CI, 0.99-3.77, $\mathrm{P}=0.04$ ) was an independent risk factor for overall survival in patients with HCC. In conclusion, SLC17A9 can be used as a new molecular marker to predict the poor prognosis of patients with HCC.
\end{abstract}

\section{Introduction}

Hepatocellular carcinoma (HCC) is a common cause of cancer-related deaths worldwide, causing approximately 740,000 deaths each year; it is the second most common cause of cancer related-deaths in the world, second only to lung

Correspondence to: Dr Jiansheng Song, Department of Emergency Surgery, The Central Hospital of Wuhan, Tongji Medical College, Huazhong University of Science and Technology, 26 Shengli Street, Wuhan, Hebei 430010, P.R. China

E-mail: sa783v@163.com

${ }^{*}$ Contributed equally

Key words: hepatocellular carcinoma, prognosis, molecular marker, SLC17A9, immunohistochemistry cancer (1). Although the diagnosis and treatment techniques of HCC are improving $(2,3)$, the most widely used treatment is hepatectomy, however approximately $70 \%$ of $\mathrm{HCC}$ patients experience postoperative recurrence and metastasis. Postoperative tumor recurrence and metastasis or chronic liver disease such as hepatitis and/or cirrhosis complications often lead to poor prognosis, especially when the tumor progresses to the advanced stage; its 5-year survival rate is $<20 \%$ and the currently available drug treatment can only bring negligible survival benefits, and the cost is high (4-6). China has a high rate of chronic hepatitis $\mathrm{B}$ virus (HBV) infection, and $\mathrm{HCC}$ patients account for nearly $55 \%$ of all cases in the world (7). Recent studies have revealed that up to $80 \%$ of the occurrence of HCC is related to chronic inflammation caused by chronic liver diseases such as hepatitis, alcoholic or non-alcoholic steatohepatitis, and may be involved in the triggering, malignant transformation, invasion and metastasis of cancer in HCC patients $(8,9)$. Solute carrier family 17 member 9 (SLC17A9) is also known as vesicular nucleotide transporter. It is a cell membrane transporter encoded by the gene located on 20q13.33, and belongs to one of the four identified new members of the solute carrier family 17 (SLC-17). It is widely expressed in human organs, tissues and cells, especially in the brain, adrenal glands and thyroid (10). Its main function is to participate in vesicle uptake, storage and secretion of adenosine triphosphate (ATP) as well as other nucleotides, and especially plays an important role in the ATP transport of airway epithelium and neutrophils, astrocytes, adrenal chromaffin cells and pancreatic cells (11). SLC17A9 can mediate ATP accumulation in lysosomes and is an indispensable factor for maintaining lysosomal physiology and cell viability (12). Studies have confirmed that SLC17A9 promotes abnormal amplification of chromosome 20q DNA, thereby driving the transformation from colorectal adenoma to colorectal cancer (13). SLC17A9 is important for cell transport and cell vitality, especially for cancer cell ATP transport (14). However, little is known about the role of SLC17A9 in HCC. The purpose of the present study was to detect the expression pattern of SLC17A9 in HCC and to elucidate its clinical significance.

\section{Materials and methods}

Standards for patient selection and inclusion. Ninety-eight patients with HCC were included in the present study, 
including 60 males and 38 females, aged 25 to 90 years old, with a median age of 57.5 years. They were treated at our hospital from January 2010 to December 2014. There were 10 cases in grade I (Edmondson classification), 50 cases in grade II and 38 cases in grade III. There were 20 cases with metastasis and 58 cases with cirrhosis. Inclusion criteria were as follows: i) The patients were diagnosed with primary HCC by pathology; ii) the patients did not undergo any treatment before surgery, including radiotherapy, chemotherapy, biotherapy or embolism; iii) the patients had complete clinical data and follow-up information; iv) in addition to HCC, the patient did not have tumors at other sites. Exclusion criteria: Those who did not meet the aforementioned standards were excluded. The present study was approved by the Ethics Committee of the Central Hospital of Wuhan (Tongji Medical College, Huazhong University of Science and Technology). Signed written informed consents were obtained from the patients and/or guardians.

Follow-up of patients. The follow-up was carried out through regular outpatient visits and telephone, and the main end-point events were recurrence and death. The survival time was from the date of surgery to the end of follow-up (August 2019) or the date of death. Tumor-free survival time was the time from the date of the surgery to the follow-up deadline or to the first recurrence during the follow-up. During the follow-up period, a total of 3 cases were lost, which was a cut-off value.

Immunochemistry. The steps for immunochemistry were as follows: The slices (4- $\mu \mathrm{m}$-thick) were fixed with $10 \%$ formaldehyde and embedded in paraffin, then they were heated at $60^{\circ} \mathrm{C}$ for $2 \mathrm{~h}$, dewaxed for $20 \mathrm{~min}$ in xylene twice, and rehydrated in gradient ethanol. Antigen repair was performed in citrate buffer $(\mathrm{pH} 6.0,10 \mathrm{mM})$ by microwave $\left(95^{\circ} \mathrm{C}, 15 \mathrm{~min}\right)$, and then was blocked with $3 \% \mathrm{H}_{2} \mathrm{O}_{2}$ for $15 \mathrm{~min}\left(28^{\circ} \mathrm{C}\right)$. After washing with phosphate-buffered saline (PBS), the slices were incubated with $5 \%$ bovine serum (Gene Tech) to block the nonspecific binding, and the anti-SLC17A9 antibody (dilution 1:100, EMD Millipore, cat. no. 2020-467092) was incubated at $4^{\circ} \mathrm{C}$ overnight. After washing three times, the tissue slices were treated with horseradish peroxidase coupled double antibody (dilution 1:300, Gene Tech, cat. no. 2017-416029) at room temperature for $30 \mathrm{~min}$ according to the manufacturer's instructions. Finally, the slices were immersed in 3,3-o-diamino-benzidine (Gene Tech) for $2 \mathrm{~min}$, stained with hematoxylin, dehydrated and observed under a microscope (Olympus BX51, x400 magnification).

Immunohistochemistry score and patient grouping. The pathological tissue sections were evaluated by two pathologists under double-blind method, and the staining score was multiplied by the staining intensity and the percentage of positive cells. The staining intensity was assessed (15) as follows: Negative staining (score 0), weak staining (score, 1), moderate staining (score 2), and strong staining (score, 3 ). The percentage of positive cells were scored as follows: $0-10 \%$ (score 0 ), $1125 \%$ (score 1), $2650 \%$ (score 2), and $51-100 \%$ (score 3). According to the results of the immunohistochemistry score, HCC patients were divided into two groups: The patients whose immunohistochemistry score was $>6$ were divided into the SLC17A9-high expression group, and the patients whose immunohistochemistry score was $\leq 6$ were divided into the SLC17A9-low expression group.

Statistical analysis. All statistical analysis was carried out using social science statistical software package (version 19.0; SPSS, Inc.). The relevance between SLC17A9 expression and clinicopathological parameters of HCC patients was analyzed by $\chi^{2}$ or Fisher's accurate test. Kaplan-Meier method and log-rank test were used to compare the survival rate of patients with high and low expression of SLC17A9. Cox proportional risk regression model was used to analyze the risk factors affecting the prognosis of patients with HCC. A P-value $<0.05$ indicated a statistically significant difference.

\section{Results}

Expression of SLC17A9 in cancer tissues of patients with HCC. Immunohistochemistry revealed that SLC17A9 was mainly expressed in the cytoplasm and partially expressed in the nucleus. Among the 98 patients with HCC, 43 (43/98, 43.88\%) had low expression of SLC17A9 and 55 (55/98, $56.12 \%$ ) had high expression of SLC17A9. The typical immunohistochemical images of SLC17A9 expression in cancer tissues of patients with $\mathrm{HCC}$ is presented in Fig. 1.

Relevance between SLC17A9 expression in cancer tissues of HCC patients and clinicopathological parameters. According to the histochemical score and the expression level of SLC17A9, patients were divided into two groups with high and low expression of SLC17A9. The expression of SLC17A9 was associated with Edmondson grade $(\mathrm{P}=0.04)$ and distant metastasis $(\mathrm{P}=0.03)$. The expression of SLC17A9 was not associated with age, sex, tumor size, tumor number, liver cirrhosis, liver cell surface antigen (HBs antigen) and AFP concentration $(\mathrm{P}>0.05$; Table I).

Association between the expression of SLC17A9 and the prognosis of HCC patients. Kaplan-Meier survival analysis revealed that the average survival time of patients with low expression of SLC17A9 was $41.12 \pm 2.78$ months. The average survival time of patients with high expression of SLC17A9 was $29.18 \pm 4.36$ months. The 5 -year tumor-free survival rate and total survival rate in patients with low expression of SLC17A9 were 24.7 and $30.7 \%$, respectively. The 5-year tumor-free survival rate and the overall survival rate in patients with high expression of SLC17A9 were 14.5 and $22.8 \%$, respectively. The tumor-free survival rate $(\mathrm{P}=0.03)$ and overall survival rate $(\mathrm{P}=0.01)$ of patients in the SLC17A9-high expression group were significantly lower than those in the SLC17A9-low expression group (Fig. 2).

Univariate and multivariate analysis affecting the tumor-free survival of patients with HCC. Univariate analysis revealed that there were several factors affecting the tumor-free survival rate of HCC patients, including tumor size $(\mathrm{P}=0.03)$, Edmondson grade $(\mathrm{P}=0.007)$, distant metastasis $(\mathrm{P}=0.006)$, microvascular invasion $(\mathrm{P}=0.02)$, the AFP level $(\mathrm{P}=0.003)$ and SLC17A9 expression $(\mathrm{P}=0.03)$. Multivariate analysis revealed 
Table I. Associations between the expression of SLC17A9 in HCC tissues and clinical parameters.

\begin{tabular}{|c|c|c|c|c|c|}
\hline \multirow[b]{2}{*}{ Clinical parameters } & \multirow[b]{2}{*}{ No. of cases } & \multicolumn{2}{|c|}{ Expression of SLC17A9 } & \multirow[b]{2}{*}{$\chi^{2}$} & \multirow[b]{2}{*}{ P-value } \\
\hline & & Low expression $(\%)$ & High expression (\%) & & \\
\hline Age (years) & & 43 & 55 & 0.32 & 0.57 \\
\hline$<55$ & 42 & 21 & 21 & & \\
\hline$\geq 55$ & 56 & 22 & 34 & & \\
\hline Sex & & & & 0.71 & 0.40 \\
\hline Male & 60 & 30 & 30 & & \\
\hline Female & 38 & 13 & 25 & & \\
\hline Tumor size $(\mathrm{cm})$ & & & & 3.28 & 0.07 \\
\hline$<5$ & 58 & 18 & 40 & & \\
\hline$\geq 5$ & 40 & 25 & 15 & & \\
\hline No. of tumors & & & & 0.14 & 0.71 \\
\hline Single tumor & 70 & 31 & 39 & & \\
\hline Multiple tumors & 28 & 12 & 16 & & \\
\hline Edmondson grade & & & & 6.38 & 0.04 \\
\hline I & 10 & 3 & 7 & & \\
\hline II & 50 & 17 & 33 & & \\
\hline III & 38 & 23 & 15 & & \\
\hline Distant metastasis & & & & 4.14 & 0.03 \\
\hline No & 78 & 33 & 45 & & \\
\hline Yes & 20 & 10 & 10 & & \\
\hline Cirrhosis & & & & 0.72 & 0.39 \\
\hline No & 40 & 21 & 19 & & \\
\hline Yes & 58 & 22 & 36 & & \\
\hline HBs antigen & & & & 0.77 & 0.29 \\
\hline Positive & 39 & 20 & 19 & & \\
\hline Negative & 59 & 23 & 36 & & \\
\hline AFP concentration & & & & 0.19 & 0.68 \\
\hline$\leq 400 \mu \mathrm{g} / \mathrm{l}$ & 29 & 13 & 16 & & \\
\hline$>400 \mu \mathrm{g} / 1$ & 69 & 30 & 39 & & \\
\hline
\end{tabular}

SLC17A9, solute carrier family 17 member 9; HCC, hepatocellular carcinoma; AFP, alpha-fetoprotein.
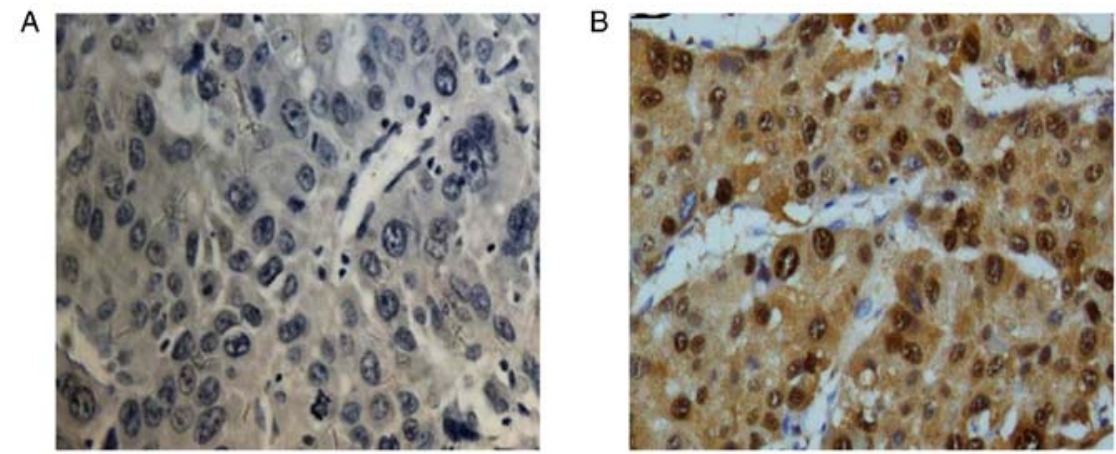

Figure 1. Typical immunohistochemical images of SLC17A9 expression in cancer tissues of HCC patients (x400). (A) Low expression of SLC17A9 in cancer tissues ( $n=43$ ). (B) High expression of SLC17A9 in cancer tissues ( $n=55$ ). SLC17A9, solute carrier family 17 member 9; HCC, hepatocellular carcinoma.

that the expression of SLC17A9 (HR, 0.77; 95\% CI, 0.27-2.47; $\mathrm{P}=0.02)$ was an independent risk factor for tumor-free survival in patients with HCC (Table II).
Univariate and multivariate analysis affecting the overall survival rate of patients with HCC. Univariate analysis revealed that the overall survival rate of patients with HCC 
Table II. Univariate and multivariate analysis of clinical parameters affecting the tumor-free survival of patients with HCC.

\begin{tabular}{|c|c|c|c|c|c|c|}
\hline \multirow[b]{2}{*}{ Clinical parameters } & \multicolumn{3}{|c|}{ Univariate analysis } & \multicolumn{3}{|c|}{ Multivariate analysis } \\
\hline & HR & $95 \% \mathrm{CI}$ & P-value & HR & $95 \% \mathrm{CI}$ & $\mathrm{P}$-value \\
\hline Age & 0.86 & $0.44-1.81$ & 0.06 & NA & & \\
\hline Sex & 1.79 & $0.87-2.49$ & 0.08 & NA & & \\
\hline Tumor size & 2.16 & $1.44-3.87$ & $0.03^{\mathrm{a}}$ & 1.56 & $0.88-3.90$ & 0.79 \\
\hline No. of tumors & 1.45 & $0.67-2.34$ & 0.51 & NA & & \\
\hline Edmondson grade & 2.77 & $2.71-4.95$ & $0.007^{\mathrm{a}}$ & 2.44 & $1.78-4.93$ & 0.02 \\
\hline Distant metastasis & 5.03 & $2.85-9.41$ & $0.006^{\mathrm{a}}$ & 3.99 & $1.27-7.64$ & 0.04 \\
\hline Microvascular infiltration & 2.32 & $1.45-3.99$ & $0.02^{\mathrm{a}}$ & 1.35 & $0.95-3.80$ & 0.39 \\
\hline HBs antigen & 1.38 & $0.75-2.88$ & 0.60 & NA & & \\
\hline Cirrhosis & 1.36 & $0.50-1.81$ & 0.58 & NA & & \\
\hline AFP level & 2.72 & $1.47-4.89$ & $0.003^{\mathrm{a}}$ & 1.83 & $0.77-4.89$ & 0.19 \\
\hline SLC17A9 expression & 0.80 & $0.26-0.89$ & $0.03^{\mathrm{a}}$ & 0.77 & $0.27-2.47$ & 0.02 \\
\hline
\end{tabular}

${ }^{\text {aP }}<0.05$. HCC, hepatocellular carcinoma; AFP, alpha-fetoprotein; SLC17A9, solute carrier family 17 member 9; HR, hazard ratio; CI, confidence interval; NA, not applicable.
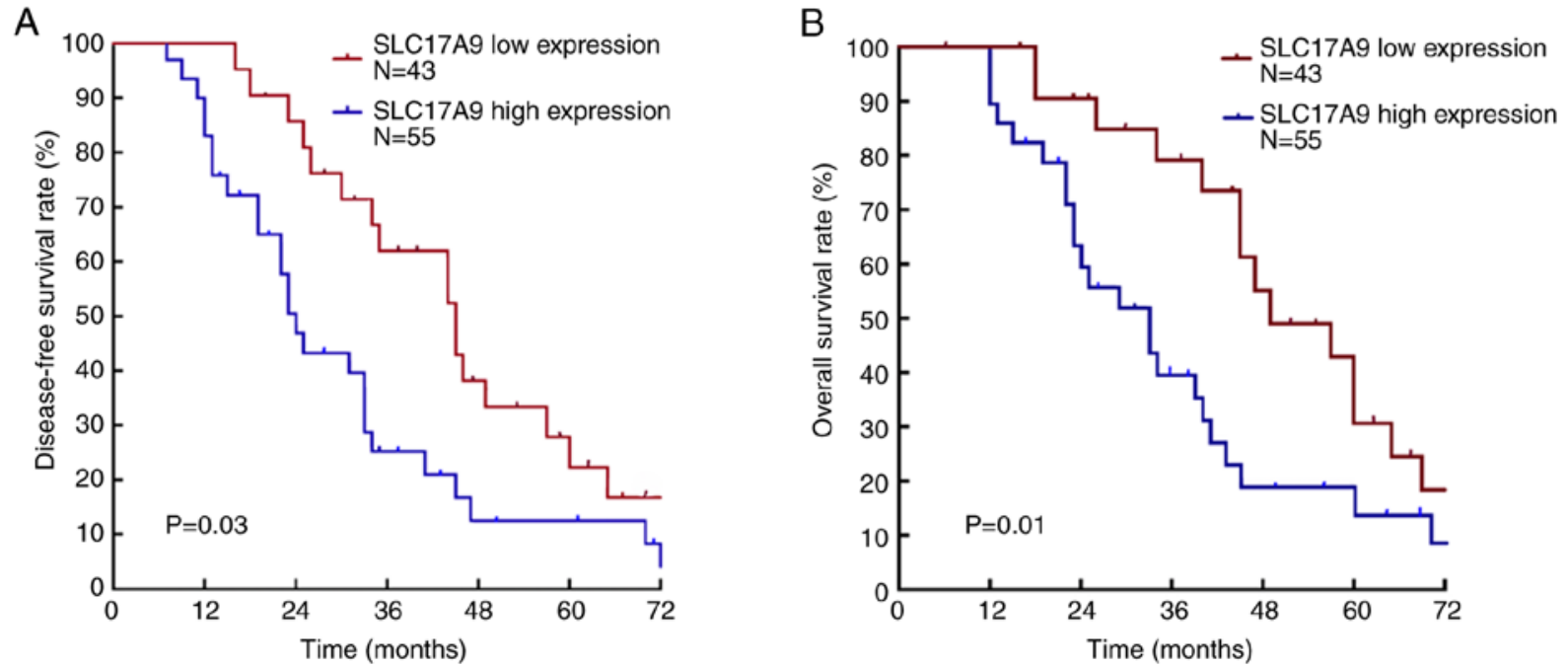

Figure 2. Comparison of (A) the tumor-free survival rate and (B) the overall survival rate in HCC patients with high and low expression of SLC17A9. HCC, hepatocellular carcinoma; SLC17A9, solute carrier family 17 member 9.

was significantly correlated with tumor size $(\mathrm{P}=0.01)$, Edmondson grade $(\mathrm{P}<0.01)$, distant metastasis $(\mathrm{P}<0.01)$, AFP level $(\mathrm{P}<0.01)$, microvascular invasion $(\mathrm{P}=0.01)$ and SLC17A9 expression $(\mathrm{P}=0.03)$. Multivariate analysis revealed that the expression of SLC17A9 (HR, 1.81; 95\% CI, 0.99-3.77; P=0.04), Edmondson grade $(\mathrm{P}=0.01)$ and distant metastasis $(\mathrm{P}=0.04)$ were independent risk factors for overall survival rate in patients with HCC (Table III).

\section{Discussion}

The incidence of HCC is ranked fifth worldwide and the mortality rate is ranked third (6). The mortality incidence of HCC in China is 383,000 people/year, and 70\% of new cases originate from Asia (7). The incidence of $\mathrm{HCC}$ is on the rise and increases steadily at a relative rate of $3 \%$ per year $(1,8)$.
Hepatectomy is the main method for the treatment of HCC, but there are still challenges to overcome such as improving the rate of radical resection and reducing the recurrence rate after surgery (8). It is particularly important to explore the biomarkers related to the prognosis of patients with HCC.

SLC17A9 is a new member of the transmembrane protein family involved in the transport of small molecules (16), which is located in the functional gene coding of human chromosome 20q13.33, in which gene mutations occur in autosomal dominant disseminated superficial hyperkeratosis patients (17). SLC17A9 is a vesicular nucleotide transporter that plays a role in ATP transport through the secretory vesicles/granule membranes of astrocytes, T cells and pancreatic cells (18). Takai et al (19) reported that knockdown of SLC17A9 could inhibit the exocytosis of ATP and reduce cell migration of human lung cancer cells. SLC17A9 protein can also function as a lysosomal ATP 
Table III. Univariate and multivariate analysis of clinical parameters affecting the overall survival rate of patients with HCC.

\begin{tabular}{|c|c|c|c|c|c|c|}
\hline \multirow[b]{2}{*}{ Clinical parameters } & \multicolumn{3}{|c|}{ Univariate analysis } & \multicolumn{3}{|c|}{ Multivariate analysis } \\
\hline & HR & $95 \% \mathrm{CI}$ & P-value & HR & $95 \% \mathrm{CI}$ & P-value \\
\hline Age & 0.65 & $0.41-1.31$ & 0.07 & NA & & \\
\hline Sex & 1.58 & $0.92-2.69$ & 0.09 & NA & & \\
\hline Tumor size & 1.95 & $1.22-3.31$ & 0.01 & 1.06 & $0.49-2.28$ & 0.89 \\
\hline No. of tumors & 1.24 & $0.68-2.26$ & 0.49 & NA & & \\
\hline Edmondson grade & 2.56 & $1.71-3.84$ & $<0.01$ & 2.44 & $1.28-4.63$ & 0.01 \\
\hline Distant metastasis & 4.82 & $2.55-9.11$ & $<0.01$ & 2.99 & $1.07-8.35$ & 0.04 \\
\hline Microvascular infiltration & 2.11 & $1.25-3.56$ & 0.01 & 1.35 & $0.65-2.80$ & 0.42 \\
\hline HBs antigen & 1.17 & $0.65-2.10$ & 0.60 & NA & & \\
\hline Cirrhosis & 1.15 & $0.70-1.91$ & 0.58 & NA & & \\
\hline AFP level & 2.51 & $1.40-4.52$ & $<0.01$ & 1.72 & $0.83-3.56$ & 0.14 \\
\hline SLC17A9 expression & 0.59 & $0.36-0.96$ & 0.03 & 1.81 & $0.99-3.77$ & 0.04 \\
\hline
\end{tabular}

HCC, hepatocellular carcinoma; AFP, alpha-fetoprotein; SLC17A9, solute carrier family 17 member 9; HR, hazard ratio; CI, confidence interval; NA, not applicable.

transporter and regulate cell viability (10). A recent study reported (20) that SLC17A9 was highly expressed in colorectal cancer. Western blotting and immunohistochemical examination revealed that the expression of SLC17A9 in cancer tissue of patients with rectal cancer was significantly higher than that of adjacent tissues, and was significantly related to the poor prognosis of patients. To date, few studies (19-21) have reported the importance of SLC17A9 in HCC.

The present study revealed that SLC17A9 was mainly located in the cytoplasm. Among the 98 patients with HCC, 43 patients $(43 / 98,43.88 \%)$ had low expression of SLC17A9 and 55 patients $(55 / 98,56.12 \%)$ had high expression of SLC17A9. A previous study reported that (20) in all 144 patients with colorectal cancer, 61 patients had low expression of SLC17A9 and 83 patients had high expression of SLC17A9, similar to the results of the present study, indicating that the low expression of SLC17A9 exhibits differential expression in different cancer tissues.

In the present study, it was revealed that the expression of SLC17A9 was associated with Edmondson grade $(\mathrm{P}=0.04)$ and distant metastasis $(\mathrm{P}=0.03)$. Results revealed that patients with high expression of SLC17A9 had higher Edmondson grade and higher rate of distant metastasis, which may be related to tumor invasiveness and metastasis of HCC, however the expression of SLC17A9 was not related to age, sex, tumor size, tumor number and liver cirrhosis $(\mathrm{P}>0.05)$. At present, it has rarely been reported that the expression of SLC17A9 is associated with the clinicopathological parameters of cancer patients $(20,21)$. It has previously been revealed that the high expression of SLC17A9 was closely related to the TNM stage of cancer patients in oral squamous cell carcinoma (21).

In the present study, Kaplan-Meier survival analysis revealed that the tumor-free survival rate $(\mathrm{P}=0.03)$ and overall survival rate $(\mathrm{P}=0.01)$ in patients with high expression of SLC17A9 were significantly lower than those in patients with low expression of SLC17A9. Multivariate analysis of Cox proportional risk model revealed that SLC17A9 expression was an independent risk factor for predicting postoperative tumor-free survival rate and overall survival rate in patients with HCC. These results indicated that SLC17A9 expression is a new biomarker for potentially predictable prognosis in patients with HCC. The association between SLC17A9 expression and cancer prognosis was limited to two different types of tumors $(20,21)$. The high expression of SLC17A9 in patients with CRC was related to the poor prognosis of patients with colorectal cancer. After grouping the patients with colorectal cancer according to different TNM stages, it was also revealed that the high expression of SLC17A9 remained an independent risk factor for predicting the poor prognosis of colorectal cancer in stage IV+III and stage I+II (20), and the survival rate of patients with high expression of SLC17A9 was significantly decreased in patients with gastric cancer (21).

The mechanism of how SLC17A9 expression affect the prognosis of patients with HCC is still not clear. Studies have reported that SLC17A9 protein is highly enriched in lysosomes and acts as an ATP transporter. SLC17A9 deficiency has been revealed to reduce lysosomal ATP accumulation and impair lysosomal function $(22,23)$. ATP release through lysosomal exocytosis was revealed to be related to cell migration $(24,25)$. In animal experiments in mice, it was determined that the high expression of SLC17A9 could promote the formation of tumor buds in colorectal cancer (25). A tumor bud is defined as a cluster of single cells or dedifferentiated tumor cells at the invasion front, which is highly correlated with epithelial-stroma transformation in tumor progression (25). In both colorectal cancer (26) and oral squamous cell carcinoma (25), the formation of tumor buds was closely related to the occurrence and progression of squamous cell carcinoma and its EMT process. In the present study, it was revealed that the high expression of SLC17A9 was related to the poor prognosis of patients with $\mathrm{HCC}$, and the poor prognosis may be due to the promotion of the progression of HCC through the epithelial-mesenchymal transition (EMT) pathway. In future studies, the molecular mechanism of the function of SLC17A9 
in patients with $\mathrm{HCC}$ will be explored. In gastric cancer patients, a previous study revealed that the expression levels of SLC17A9 mRNA and protein in cancer tissues of gastric cancer patients were significantly higher than those in adjacent tissues; the high expression of SLC17A9 protein was positively correlated with the mutation of tumor protein p53 gene (TP53) in cancer tissues of patients, and was an independent risk factor for postoperative recurrence and mortality prediction of the patients and thus it was suggested that SLC17A9 may drive TP53 gene mutations and participate in gastric cancer progression and poor prognosis (21). A recent genome-wide sequence analysis of 130 patients with acute lymphoblastic leukemia (including 69 adult patients and 61 pediatric patients) also revealed aberrant expression of SLC17A9 mRNA; SLC17A9 mRNA overexpression was an independent risk factor of the poor prognosis for adult patients with acute lymphoblastic leukemia, and no association between SLC17A9 mRNA and patient prognosis was found in pediatric patients (27). The present study revealed that in patients with HCC, patients with high SLC17A9 protein expression had a poor prognosis, and its molecular mechanism remains to be further explored.

The limitations of the present study include: First, the study was a single-center retrospective study, and the number of included cases was small; large-scale multicenter prospective studies are required to further confirm the conclusions of the present study. Second, the molecular mechanism of SLC17A9 function in patients with $\mathrm{HCC}$ requires further study.

In conclusion, the results of the present study revealed that the prognosis of patients with high expression of SLC17A9 in HCC patients was significantly lower than that of patients with low expression of SLC17A9. The high expression of SLC17A9 may play an important role in the progression of $\mathrm{HCC}$, which may be a new prognostic molecular marker and potential therapeutic target in patients with HCC.

\section{Acknowledgements}

Not applicable.

\section{Funding}

No funding was received.

\section{Availability of data and materials}

The datasets used and/or analyzed during the present study are available from the corresponding author on reasonable request.

\section{Authors' contributions}

JW and YY conceived and designed the study. JW, YY and JS were responsible for the collection, analysis and interpretation of the data. JW drafted the manuscript. YY revised the manuscript critically for important intellectual content. All authors read and approved the final manuscript.

\section{Ethics approval and consent to participate}

The present study was approved by the Ethics Committee of The Central Hospital of Wuhan, Tongji Medical College,
Huazhong University of Science and Technology. Signed written informed consents were obtained from the patients and/or guardians.

\section{Patient consent for publication}

Not applicable.

\section{Competing interests}

The authors declare that they have no competing interests.

\section{References}

1. Torre LA, Bray F, Siegel RL, Ferlay J, Lortet-Tieulent J and Jemal A: Global cancer statistics, 2012. CA Cancer J Clin 65: 87-108, 2015.

2. Lee S, Kang TW, Cha DI, Song KD, Lee MW, Rhim H, Lim HK, Sinn DH, Kim JM and Kim K: Radiofrequency ablation vs. surgery for perivascular hepatocellular carcinoma: Propensity score analyses of long-term outcomes. J Hepatol 69: 70-78, 2018.

3. Romano A, Angeli P, Piovesan S, Noventa F, Anastassopoulos G, Chemello L, Cavalletto L, Gambato M, Russo FP, Burra P, et al: Newly diagnosed hepatocellular carcinoma in patients with advanced hepatitis $C$ treated with DAAs: A prospective population study. J Hepatol 69: 345-352, 2018.

4. Tokumitsu Y, Sakamoto K, Tokuhisa Y, Matsui H, Matsukuma S, Maeda Y, Sakata K, Wada H, Eguchi H, Ogihara H, et al: A new prognostic model for hepatocellular carcinoma recurrence after curative hepatectomy. Oncol Lett 15: 4411-4422, 2018.

5. Zhu Q, Li N, Zeng X, Han Q, Li F, Yang C, Lv Y, Zhou Z and Liu Z: Hepatocellular carcinoma in a large medical center of China over a 10-year period: Evolving therapeutic option and improving survival. Oncotarget 6: 4440, 2015.

6. Parikh ND, Singal AG and Hutton DW: Cost effectiveness of regorafenib as second-line therapy for patients with advanced hepatocellular carcinoma. Cancer 123: 3725-3731, 2017.

7. Yang JD, Hainaut P, Gores GJ, Amadou A, Plymoth A and Roberts LR: A global view of hepatocellular carcinoma: Trends, risk, prevention and management. Nat Rev Gastroenterol Hepatol 22: 589-604, 2019.

8. Zhu RX, Seto WK, Lai CL and Yuen MF: Epidemiology of hepatocellular carcinoma in the Asia-Pacific region. Gut Liver 10: 332, 2016.

9. Levrero M and Zucman-Rossi J: Mechanisms of HBV-induced hepatocellular carcinoma. J Hepatol 64 (Suppl 1): S84-S101, 2016.

10. Sreedharan S, Shaik JH, Olszewski PK, Levine AS, Schiöth HB and Fredriksson R: Glutamate, aspartate and nucleotide transporters in the SLC17 family form four main phylogenetic clusters: Evolution and tissue expression. BMC Genomics 11: 17, 2010.

11. Bissa B, Beedle AM and Govindarajan R: Lysosomal solute carrier transporters gain momentum in research. Clin Pharmacol Ther 100: 431-436, 2016.

12. Moriyama Y, Hiasa M, Sakamoto S, Omote $\mathrm{H}$ and Nomura $\mathrm{M}$ : Vesicular nucleotide transporter (VNUT): Appearance of an actress on the stage of purinergic signaling. Purinergic Signal 13: 387-404, 2017.

13. Cao Q, Zhao K, Zhong XZ, Zou Y, Yu H, Huang P, Xu TL and Dong XP: SLC17A9 protein functions as a lysosomal ATP transporter and regulates cell viability. J Biol Chem 289: 23189-23199, 2014.

14. Sillars-Hardebol AH, Carvalho B, Tijssen M, Beliën JA, de Wit M, Delis-van Diemen PM, Pontén F, van de Wiel MA, Fijneman RJ and Meijer GA: TPX2 and AURKA promote 20q Amplicon-driven colorectal adenoma to carcinoma progression. Gut 61: 1568-1575, 2012.

15. Weinberg SE and Chandel NS: Targeting mitochondria metabolism for cancer therapy. Nat Chem Biol 11: 9, 2015.

16. Zhai E, Liang W, Lin Y, Huang L, He X, Cai S, Chen J, Zhang N, Li J, Zhang Q, et al: HSP70/HSP90-organizing protein contributes to gastric Cancer progression in an autocrine fashion and predicts poor survival in gastric Cancer. Cell Physiol Biochem 47: 879-892, 2018 
17. Cui H, Li L, Wang W, Shen J, Yue Z, Zheng X, Zuo X, Liang B, Gao M, Fan X, et al: Exome sequencing identifies SLC17A9 pathogenic gene in two Chinese pedigrees with disseminated superficial actinic porokeratosis. J Med Genet 51: 699-704, 2014.

18. Dereure O: Mutation in the SLC17A9 gene in familial superficial actinic disseminated porokeratosis. Ann Dermatol Venereol 142: 155-156, 2015 (In French).

19. Takai E, Tsukimoto M, Harada H, Sawada K, Moriyama Y and Kojima S: Autocrine regulation of TGF- $\beta 1$-induced cell migration by exocytosis of ATP and activation of $\mathrm{P} 2$ receptors in human lung cancer cells. J Cell Sci 125: 5051-5060, 2012.

20. Yang L, Chen Z, Xiong W, Ren H, Zhai E, Xu K, Yang H, Zhang Z, Ding L, He Y, et al: High expression of SLC17A9 correlates with poor prognosis in colorectal cancer. Human Pathol 84 62-70, 2019.

21. Li J, Su T, Yang L, Deng L, Zhang C and He Y: High SLC17A9 expression correlates with poor survival in gastric carcinoma. Future Oncol 15: 4155-4166, 2019.

22. Haanes KA, Kowal JM, Arpino G, Lange SC, Moriyama Y, Pedersen PA and Novak I: Role of vesicular nucleotide transporter VNUT (SLC17A9) in release of ATP from AR42J cells and mouse pancreatic acinar cells. Purinergic Signal 10: 431-440, 2014.

23. Koike M, Nakanishi H, Saftig P, Ezaki J, Isahara K, Ohsawa Y, Schulz-Schaeffer W, Watanabe T, Waguri S, Kametaka S, et al: Cathepsin D deficiency induces lysosomal storage with ceroid lipofuscin in mouse CNS neurons. J Neurosci 20: 6898-6906, 2000
24. Dou Y, Wu HJ, Li HQ, Qin S, Wang YE, Li J, Lou HF, Chen Z, Li XM, Luo QM and Duan S: Microglial migration mediated by ATP-induced ATP release from lysosomes. Cell Res 22: 1022-1033, 2012.

25. Hong KO, Oh KY, Shin WJ, Yoon HJ, Lee JI and Hong SD: Tumor budding is associated with poor prognosis of oral squamous cell carcinoma and histologically represents an epithelial-mesenchymal transition process. Hum Pathol 80: 123-129, 2018.

26. De Smedt L, Palmans S, Andel D, Govaere O, Boeckx B, Smeets D, Galle E, Wouters J, Barras D, Suffiotti M, et al: Expression profiling of budding cells in colorectal cancer reveals an EMT-like phenotype and molecular subtype switching. $\mathrm{Br}$ J Cancer 116: 58-65, 2017

27. Chen B, Jiang L, Zhong ML, Li JF, Li BS, Peng LJ, Dai YT, Cui BW, Yan TQ, Zhang WN, et al: Identification of fusion genes and characterization of transcriptome features in T-cell acute lymphoblastic leukemia. Proc Natl Acad Sci USA 115: 373-378, 2018.

cc) (i) $\ominus$ This work is licensed under a Creative Commons Attribution-NonCommercial-NoDerivatives 4.0 International (CC BY-NC-ND 4.0) License. 